\title{
Study of Orientational Order of Liquid Crystal 8 OCB Doped with Perylene-like Dyes by Means of Polarized Optical Spectroscopy
}

\author{
Danuta Bauman and Eryk Wolarz \\ Institute of Physics, Poznań University of Technology, Piotrowo 3, 60-965 Poznań, Poland
}

Z. Naturforsch. 51 a, 1192-1196 (1996); received October 5, 1996

\begin{abstract}
Measurements of the polarized absorption and fluorescence spectra for perylene-like dyes dissolved in a liquid crystal $8 \mathrm{OCB}$ have been used to study the long-range orientational order in the smectic $\mathrm{A}$ and nematic phases. The temperature dependence of the order parameters $\left\langle P_{2}\right\rangle$ and $\left\langle P_{4}\right\rangle$ has been investigated and the orientational distribution function has been determined. On the basis of the experimental order parameter values some information about the orientation of molecules in guest-host mixtures has been obtained.
\end{abstract}

\section{Introduction}

The problem of the determination of the orientational order in dichroic dye - liquid crystal mixtures (guest-host systems) is of interest in view of the technological application of guest - host liquid crystal display devices, and also of primary importance in recognizing and understanding the intermolecular interaction in anisotropic media.

In a nematic liquid crystalline phase the molecules are, on average, aligned with their long axes parallel to each other, which leads macroscopically to a preferred direction, described by the director $\mathbf{n}$, and the centres of mass of the molecules are placed randomly. Therefore, in this phase only a long-range orientational order occurs. In smectic phases the molecular centres of mass sit on planes perpendicular to the preferred axis, leading to a layer structure, so not only orientational but also positional order occurs. For optically uniaxial smectic phases, e.g. smectic A phase, the orientational order can be evaluated from any of the methods used for the nematic phase, i.e. from the anisotropy of the refractive index, dielectric constant, diamagnetic susceptibility, or by using a wide variety of spectroscopic methods.

In this paper we have studied the long-range orientational order both in the smectic $\mathrm{A}$ and nematic phases of mixtures of 4'- $n$-octyloxy-4-n-cyanobiphenyl ( 8 OCB) with fluorescent perylene-like dyes using the polarized absorption and fluorescence spectroscopy.

Reprint requests to Prof. Danuta Bauman.

\section{Materials and Methods}

The mesogen 4'-n-octyloxy-4- $n$-cyanobiphenyl (8 OCB) was synthesized in the Laboratory of Professor R. Dabrowski at the Military Academy of Technology in Warsaw and was used without further purification. The temperatures of the phase transitions were in the agreement with the literature $[1,2]$ and were as follows:

$$
\mathrm{C}-327.7 \mathrm{~K}-\mathrm{S}_{\mathrm{A}}-340.2 \mathrm{~K}-\mathrm{N}-353.2 \mathrm{~K}-\mathrm{I} .
$$

As guest species three dichroic dyes, derivatives of 3,4,9,10-perylenetetracarboxylic acid with the following chemical structure were used:

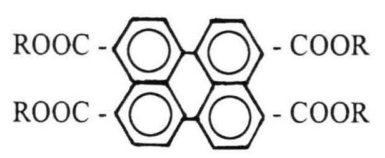

\begin{tabular}{|c|c|}
\hline Dye code & $\mathrm{R}$ \\
\hline 1 & $-\left(\mathrm{CH}_{2}\right)_{3} \mathrm{CH}_{3}$ \\
2 & $-\left(\mathrm{CH}_{2}\right)_{4} \mathrm{CH}_{3}$ \\
3 & $-\left(\mathrm{CH}_{2}\right)_{6} \mathrm{CH}_{3}$ \\
\hline
\end{tabular}

The dyes were synthesized and chromatographically purified in the Institute of Dyes, Łódź University of Technology, Poland and were dissolved in liquid crystal matrix at a concentration of $1.5 \cdot 10^{-3} \mathrm{M}$. It was found in [3] that they have brilliant yellow colour and emit green fluorescence light with very high quantum yield. 
The polarized absorption spectra were measured with a double beam spectrophotometer SPECORD M 40 (Carl Zeiss Jena, Germany). The polarized fluorescence spectra were recorded using a home-made photon-counting fluorimeter in the $\pi$ geometry, i.e. the exciting light beam was perpendicular to the cell surface and the fluorescence light was monitored perpendicularly from the same side of the cell. Both the spectrophotometer and fluorimeter were equipped with neutral UV polarizers. Corrections for the photomultiplier spectral sensitivity and for the different response of the device set with various directions of polarization were made. The measurements were carried out as functions of temperature in $20 \mu \mathrm{m}$ thick "sandwich" cells. A planar orientation of the liquid crystal and dye molecules was achieved by treating the glass surfaces of the cells with polyimide and additional rubbing. This procedure gives a good orientation of the molecules in a thin layer, which was controlled with a polarizing microscope. The temperature of the cells was regulated and controlled with an accuracy of $\pm 0.1 \mathrm{~K}$.

\section{Orientational Order in Uniaxial Liquid Crystalline Phases}

Long-range orientational order in the liquid crystalline phases arises because of the tendency of the rodlike molecules to align their long axes along the direction of the director, $\mathbf{n}$. At finite temperature the thermal motion of the molecules prevents perfect alignment with $\mathbf{n}$; the orientation of the molecules is in fact distributed in angle, but with the director as the most probable, or the most populated, direction. The orientational order of an anisotropic ensemble of molecules can be described by an orientational distribution function $f(\Omega)$, where $\Omega$ comprises the Eulerian angles $\alpha, \beta$ and $\gamma$ between the space-fixed and molecule-fixed coordinate systems. Only uniaxial symmetry around the director is characterized for many liquid crystals, thus no order in the angle $\alpha$ (rotation in the azimuthal direction) is permitted. Moreover, if one assumes that the liquid crystal molecules are both rigid and possess $\mathrm{C}_{\infty \mathrm{v}}$ symmetry, the rotation around the molecular axis (angle $\gamma$ ) should not modify the distribution function. Thus, consideration of an abbreviated orientational distribution function $f(\beta)$ is now sufficient, where $\beta$ is the angle between the director and the long axis of the liquid crystal molecule.
The distribution function $f(\beta)$ can be expanded in a series of even Legendre polynomials, $P_{2 L}(\cos \beta)$ as [4]

$$
f(\beta)=\sum_{L=0}^{\infty} \frac{4 L+1}{2}\left\langle P_{2 L}(\cos \beta)\right\rangle P_{2 L}(\cos \beta),
$$

where

$$
\left\langle P_{2 L}\right\rangle=\frac{\int_{0}^{\pi / 2} P_{2 L}(\cos \beta) f(\beta) \sin \beta \mathrm{d} \beta}{\int_{0}^{\pi / 2} f(\beta) \sin \beta \mathrm{d} \beta} .
$$

The coefficient of the first nontrivial term in the above expansion is the order parameter $\left\langle P_{2}\right\rangle=\mathrm{S}[5,6]$. The order parameter can be obtained using many techniques including optical birefringence, diamagnetic anisotropy and dichroism as well as nuclear magnetic resonance studies. Electron spin resonance, polarized Raman and fluorescence emission spectra of liquid crystals doped with suitable probes give information, in addition to $\left\langle P_{2}\right\rangle$, on the fourth-rank parameter $\left\langle P_{4}\right\rangle$. Knowledge of $\left\langle P_{2}\right\rangle$ and $\left\langle P_{4}\right\rangle$ allows to obtain the truncated distribution function, $f_{4}(\beta)$, where:

$$
f_{4}(\beta)=\frac{1}{2}+\frac{5}{2}\left\langle P_{2}\right\rangle P_{2}+\frac{9}{2}\left\langle P_{4}\right\rangle P_{4} .
$$

Experimental determination of the long-range orientational order for guest-host mixtures is usually carried out using the classical methods of optical spectroscopy. Doping the liquid crystal with a fluorescent probe and utilizing "guest-host" effect [7, 8], information about the order can be obtained both from the polarized absorption and fluorescence measurements.

On the basis of the optical absorption anisotropy, one can calculate the average of the second Legendre polynomial, $\left\langle P_{2}\right\rangle$ using the formula [9]

$$
\left\langle P_{2}\right\rangle=\frac{A_{\|}-A_{\perp}}{A_{\|}+2 A_{\perp}},
$$

where $A_{\|}$and $A_{\perp}$ are the absorbances of the light polarized parallel and perpendicular to the orientation axis of the liquid crystal, respectively.

From the fluorescence measurements, the emission anisotropies, $R_{1}$ and $R_{2}$ for excitation with light polarized, respectively, parallel and perpendicular to the director, $\mathbf{n}$ can be calculated from the formula

$$
R_{1,2}=\frac{J_{\|}+J_{\perp}}{J_{\|}+2 J_{\perp}}
$$


where $J_{\|}$and $J_{\perp}$ are the intensities of fluorescence polarized parallel and perpendicular to the director, respectively, measured in the parallel geometry. They require corrections for instrumental, concentration and volume factors.

If we assume that the rotational time, $\tau_{\mathrm{R}}$ is much longer than the lifetime $\tau_{\mathrm{F}}$ of the excited state of the fluorescent molecule, then $R_{1}$ and $R_{2}$ can be related with $\left\langle P_{2}\right\rangle$ and $\left\langle P_{4}\right\rangle[10]$ :

$$
\begin{aligned}
& R_{1}=\frac{\left[\frac{2}{5}+\frac{11}{7}\left\langle P_{2}\right\rangle+\frac{36}{35}\left\langle P_{4}\right\rangle\right] P_{2}(\cos \delta)}{1+2\left\langle P_{2}\right\rangle}, \\
& R_{2}=\frac{\left[\left\langle P_{2}\right\rangle-\frac{2}{5}-\frac{21}{35}\left\langle P_{4}\right\rangle\right] P_{2}(\cos \delta)}{1-\left\langle P_{2}\right\rangle+2\left[\frac{1}{5}-\frac{2}{7}\left\langle P_{2}\right\rangle+\frac{3}{35}\left\langle P_{4}\right\rangle\right] P_{2}(\cos \delta)} .
\end{aligned}
$$

Equations (4), (6), and (7) are valid if the angle between the vector of the absorption transition moment and the long axis of a dye molecule is $0^{\circ}$. For symmetrically substituted perylene derivatives one can assume that the absorption transition moment of the longest wavelength is polarized, similarly as in perylene [11], parallel to the long axis of the molecule.

\section{Results}

For calculation of the order parameters, $\left\langle P_{2}\right\rangle$ and $\left\langle P_{4}\right\rangle$ from the absorption and fluorescence measurements, the values of the absorbances and fluorescence intensities were taken at the wavelength corresponding to the maximum of the absorption $\left(\lambda_{\mathrm{A}}^{\max }=480 \mathrm{~nm}\right)$ and emission $\left(\lambda_{\mathrm{F}}^{\max }=500 \mathrm{~nm}\right)$ bands, respectively. The angle $\delta$ between the absorption and emission oscillators, which was needed to determine the order parameters from (6) and (7), was estimated on the basis of the absorption and fluorescence measurements of the dyes in $8 \mathrm{OCB}$ at the temperature just after crystalsmectic A transition. We have supposed that in the smectic state, because of its high viscosity, the thermal molecular motions are strongly hindered and have a negligible effect on the emission anisotropy. Therefore, in this case $\left\langle P_{2}\right\rangle$ obtained from the fluorescence measurement must be equal to that determined on the basis of the polarized absorption spectra. By knowing $\left\langle P_{2}\right\rangle$ from absorption measurement (4), $\left\langle P_{4}\right\rangle$ and $\cos \delta$ was determined by solving (6) and (7). We have ob- tained $18^{\circ} \pm 4^{\circ}$ for dye 1 and $\delta=20^{\circ} \pm 4^{\circ}$ for dyes 2 and 3. Then the angle $\delta$ was assumed to be constant in our experimental temperature range, and the order parameters $\left\langle P_{2}\right\rangle_{\mathrm{F}}$ and $\left\langle P_{4}\right\rangle_{\mathrm{F}}$ as a function of temperature were determined from the measured fluorescence intensities and (6) and (7). Additionally, $\left\langle P_{2}\right\rangle_{\mathrm{A}}$ was estimated independently from (4), using the results of the polarized absorption measurement.

Figures 1-3 present the order parameters $\left\langle P_{2}\right\rangle_{\mathrm{A}}$ (open circles), $\left\langle P_{2}\right\rangle_{\mathrm{F}}$ (filled circles) and $\left\langle P_{4}\right\rangle_{\mathrm{F}}$ (squares) versus temperature $T^{*}$ for 8 OCB doped with dyes 1 , 2 and 3 . The triangles in these figures represent the values of $\left\langle P_{2}\right\rangle$ for pure 8 OCB estimated on the basis of the optical birefringence measurement [12] using the method described in [13]. $T^{*}=T / T_{\mathrm{NI}}$ where $T_{\mathrm{NI}}$ is the clearing temperature for the 8 OCB-dye mixture or for pure $8 \mathrm{OCB}$ and $T$ is the temperature of the measurement.

In Fig. 4 the distribution functions $f_{4}(\beta)$ for $8 \mathrm{OCB}$ doped with dye 3 in the smectic $\mathrm{A}(T=329.0 \mathrm{~K})$ and nematic $(T=343.5 \mathrm{~K})$ phases are plotted.

\section{Discussion}

From results presented in Figs. $1-3$ follows that the order parameters $\left\langle P_{2}\right\rangle_{\mathrm{A}}$ coincide with $\left\langle P_{2}\right\rangle_{\mathrm{F}}$ within experimental uncertainties. Moreover, it is seen that the long-range orientational order in the guest-host mixtures changes with the temperature, but in the smectic A phase less than in the nematic one. The character of the temperature changes for all three mixtures is similar, but the values of the order parameter at a given temperature are different. The best molecular orientation is observed for the 8 OCB-dye 3 mixture. The values of $\left\langle P_{2}\right\rangle$ for this mixture in the smectic A phase are similar to those obtained for pure 8 OCB, whereas in the nematic phase they are slightly lower than for pure host. The dyes $\mathbf{1}$ and $\mathbf{2}$ have significantly worse orientation. Comparison of the results presented in Figs. 1-3 shows that the order parameter increases with the length of the dye molecule. It seems that the perylene derivatives cannot be used as fluorescent probes to study the orientational behaviour of liquid crystal matrices. From the polarized absorption and fluorescence measurements only information about the orientational order of the dye molecules dissolved in the liquid crystal can be obtained because the guest-host interactions disturbe molecular alignment of the matrix. 


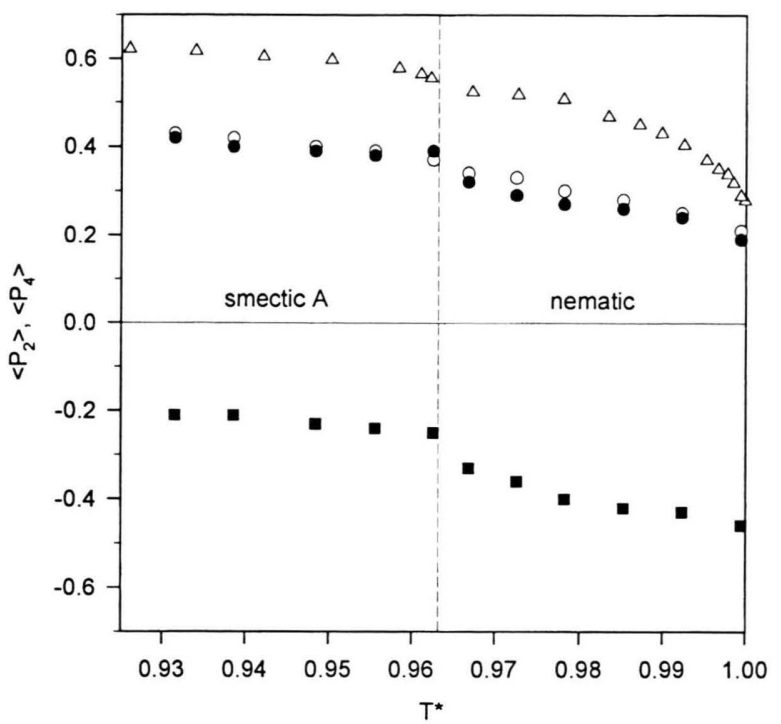

Fig. 1. $\left\langle P_{2}\right\rangle_{\mathrm{A}}$ (open circles), $\left\langle P_{2}\right\rangle_{\mathrm{F}}$ (filled circles) and $\left\langle P_{4}\right\rangle_{\mathrm{F}}$ (squares) as functions of the reduced temperature for $8 \mathrm{OCB}$ doped with dye 1 . The triangles represent the values of $\left\langle P_{2}\right\rangle$ for pure 8 OCB [12].

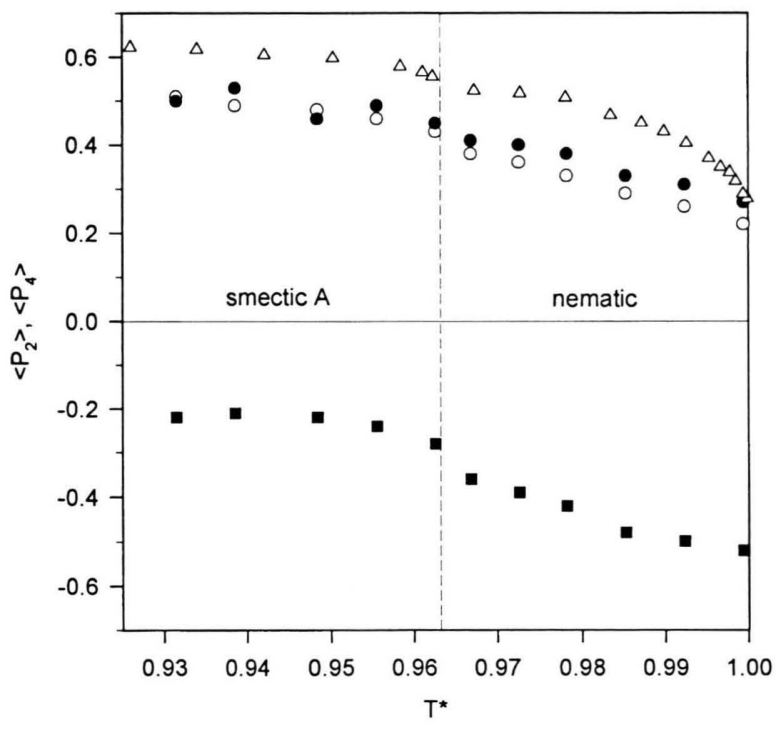

Fig. 2. $\left\langle P_{2}\right\rangle_{\mathrm{A}}$ (open circles), $\left\langle P_{2}\right\rangle_{\mathrm{F}}$ (filled circles) and $\left\langle P_{4}\right\rangle_{\mathrm{F}}$ (squares) as functions of the reduced temperature for $8 \mathrm{OCB}$ doped with dye 2 . The triangles represent the values of $\left\langle P_{2}\right\rangle$ for pure $8 \mathrm{OCB}[12]$.

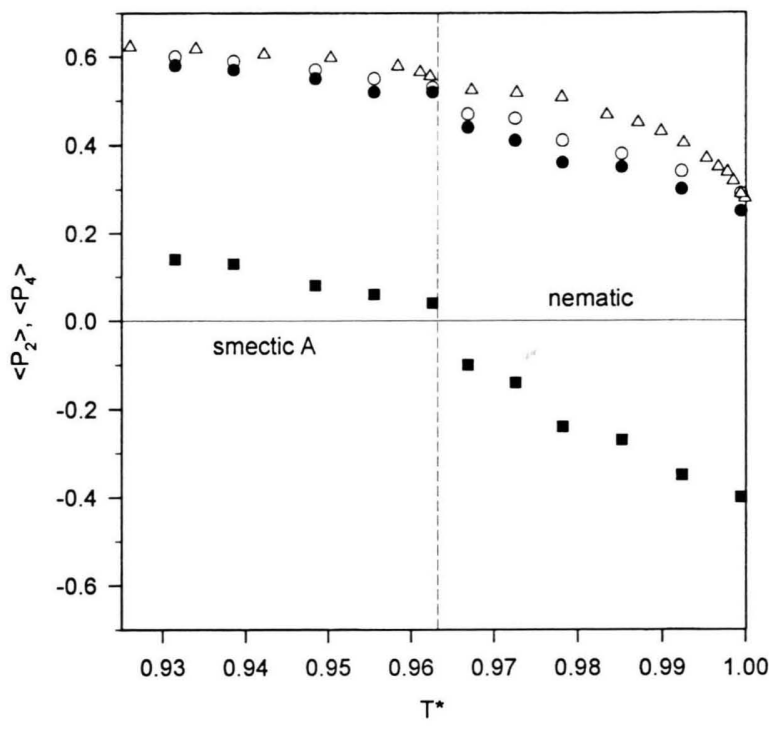

Fig. 3. $\left\langle P_{2}\right\rangle_{\mathrm{A}}$ (open circles), $\left\langle P_{2}\right\rangle_{\mathrm{F}}$ (filled circles) and $\left\langle P_{4}\right\rangle_{\mathrm{F}}$ (squares) as functions of the reduced temperature for $8 \mathrm{OCB}$ doped with dye 3. The triangles represent the values of $\left\langle P_{2}\right\rangle$ for pure $8 \mathrm{OCB}[12]$.

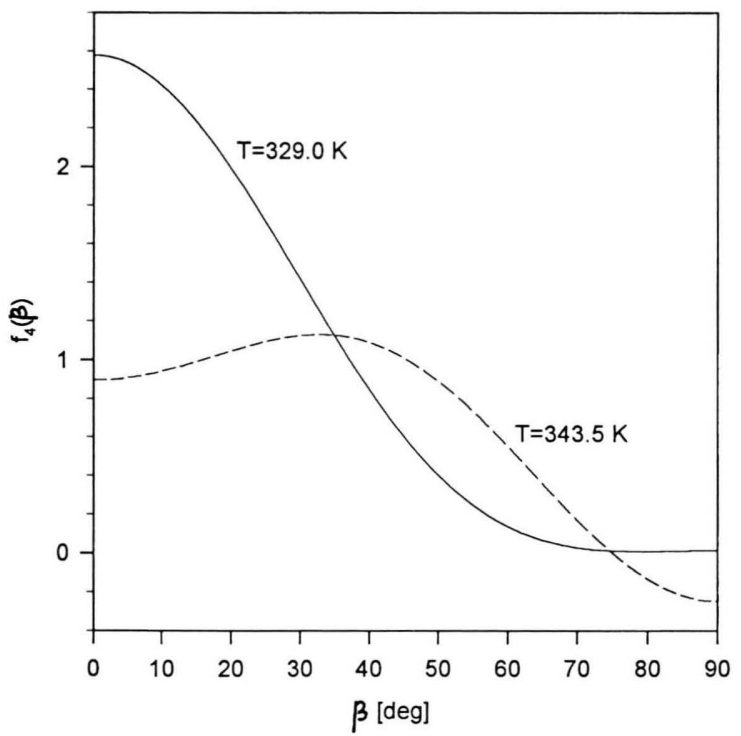

Fig. 4. Orientational distribution function $f_{4}(\beta)$ in the smectic A $(T=329.0 \mathrm{~K})$ and nematic $(T=343.5 \mathrm{~K})$ phases of the 8 OCB-dye 3 mixture. 
As regards $\left\langle P_{4}\right\rangle$, Figs. $1-3$ show that this parameter is positive only for the 8 OCB-dye 3 mixture in the smectic A phase, while it becomes negative after the $\mathrm{S}_{\mathrm{A}} \rightarrow \mathrm{N}$ transition. For $8 \mathrm{OCB}$ doped with dyes $\mathbf{1}$ and $\mathbf{2}\left\langle P_{4}\right\rangle$ is always negative, even in the smectic A phase. The order parameter $\left\langle P_{4}\right\rangle$ depends on higher powers of the deviation angle $\beta$, is therefore more sensitive to molecular fluctuations and decreases rapidly if the molecular orientation is perturbed by a guest. This behaviour of $\left\langle P_{4}\right\rangle$ was also observed for many dyeliquid crystal mixtures [4,14-19], although it is not in agreement with the theoretical predictions $[20,21]$. It seems that the low negative values of $\left\langle P_{4}\right\rangle$ obtained for the 8 OCB-dye mixtures investigated in this paper are due to the strong tendency of the dye molecules to tip away from the director, an effect which is probably connected with the molecular geometry of the

[1] G. R. Luckhurst and R. Poupko, Mol. Phys. 29, 1293 (1975).

[2] M. Mitra, Mol. Cryst. Liq. Cryst. 241, 17 (1994).

[3] E. Mykowska and D. Bauman, Z. Naturforsch. 51 a, 843 (1996).

[4] L. L. Chapoy and D. B. Du Pre, in: Liquid Crystals and Ordered Fluids, Vol. 3, ed. J. F. Johnson and R. S. Porter, Plenum Press, New York 1978.

[5] V. Tsvetkov, Acta Physicochim. USSR, 16, 1312 (1942).

[6] A. Saupe, Z. Naturforsch. 19a, 161 (1964).

[7] G. H. Heilmeier and L. A. Zanoni, Appl. Phys. Lett. 13, 91 (1968).

[8] G. H. Heilmeier, J. A. Castellano, and L. A. Zanoni, Mol. Cryst. Liq. Cryst. 8, 293 (1969).

[9] D. L. White and G. N. Taylor, J. Appl. Phys. 45, 4718 (1974).

[10] C. Zannoni, Molec. Phys. 38, 1813 (1979).

[11] J. Michl and E. W. Thulstrup, Spectroscopy with Polarized Light, VCH Publishers, Inc., New York 1986.

[12] D. Bauman and H. Moryson, J. Mol. Struct., in press. perylene derivatives, different from the geometry of the liquid crystal molecules.

The negative value of $\left\langle P_{4}\right\rangle$ influences the shape of the molecular distribution function. Figure 4 shows that $f_{4}(\beta)$ for the 8 OCB-dye 3 mixture is significantly broadened at the temperature corresponding to the nematic phase in comparison to $f_{4}(\beta)$ determined in the smectic A phase. A similar shape of the orientational distribution function was obtained by Catalano et al. [16] for perylene in the nematic lyotropic phase by using the fluorescence depolarization method.

\section{Acknowledgements}

This work was supported by Poznan University of Technology Research Project BW 62-125.

[13] B. Żywucki, W. Kuczyński, and G. Czechowski, in: Liquid Crystals: Materials Science and Applications, J. Żmija, Z. Raszewski, J. Zieliński, eds., Proc. SPIE 2372, 151 (1995).

[14] E. Wolarz and D. Bauman, Mol. Cryst. Liq. Cryst. 197, 1 (1991)

[15] D. Bauman, H. Moryson, and E. Wolarz, Acta Phys. Polon. A, 81, 559 (1992).

[16] D. Catalano, A. Lenzi, and C. A. Veracini, Liq. Cryst. 14, 1457 (1993).

[17] H. Moryson and D. Bauman, Mol. Cryst. Liq. Cryst. 250, 63 (1994).

[18] D. Bauman, T. Hanemann, and E. Wolarz, Z. Naturforsch. 49a, 671 (1994).

[19] E. Wolarz and D. Bauman, Liq. Cryst. 19, 221 (1995).

[20] W. Maier and A. Saupe, Z. Naturforsch. 13a, 5564 (1958); 14 a, 882 (1959); 15a, 287 (1960); 16a, 262 (1961).

[21] R. L. Humphries, P. G. James, and G. R. Luckhurst, J. Chem. Soc. Faraday Trans. II, 68, 1031 (1972). 